\title{
Il 'nicodemismo' di Michelangelo nei "sonetti sulla notte"
}

\author{
Joseph Francese
}

È stata notata recentemente (Saslow 26) l'estrema importanza della vista per l'io poetante michelangiolesco. Infatti il tema della cecità e del buio spirituale appare spesso nel canzoniere del Buonarroti, e quasi sempre con una connotazione negativa (cieco ardore, cieco furore, ecc.). Solo nei sonetti sulla notte (Perché Febo non torce e non distende, $O$ notte, o dolce tempo, benché nero, Ogni van chiuso, ogni coperto loco, Colui che fece, e non di cosa alcuna) si verifica un cambiamento di significato. Percio occorre considerarli un gruppo distinto, ma non omogeneo, all'interno delle liriche dedicate al Cavalieri ${ }^{1}$ ed anche, data la loro accentuata religiosità, un preludio ai componimenti scritti per Vittoria Colonna.

All'interno di questo raggruppamento, in Ogni van chiuso, ogni coperto loco si trasgredisce la metafisica ficiniana della luce. L'universal Bellezza non è rivolta agli occhi dall'esterno bensì è illuminazione interna all'individuo. La notte non è sinonimo della morte (che a sua volta significava la pace dell'anima e l'ascesa in Cielo) ma il mezzo necessario all'io poetante per attingere ad una visione privilegiata del volto e della luce divini. In questo componimento si viene a creare una tensione narrativa nella quale la fede interiore del poeta deve lottare per proteggere la sua notte idiosincratica, l'unico vivaio adatto a covare la divinità dell'uomo. In questo saggio cercheremo di dimostrare il modo in cui il 'nicodemismo' di Michelangelo, ossia la sua opposizione passiva ai dettami della Controriforma, informa questo tentativo poetico di realizzazione spirituale.

Il tema religioso si fa sentire nei versi di Michelangelo sin dalle prime opere letterarie, in un primo tempo facendo da contrappunto ad altri temi più mondani, benché il Buonarroti fosse già disposto da giovane alla riforma della Chiesa cattolica, sia nel costume, sia in questioni di dottrina. Tuttavia la frequenza e l'intensità del monologo spirituale prende impeto veramente solo dopo la conoscenza della Colonna; diventa poi motivo predominante dopo la morte di lei, avvenuta nel 1547 quando il Buonarroti aveva settantatré anni. L'influenza della Colonna su Michelangelo è ben documentata, e i versi di Michelangelo devono molto alle discussioni religiose fra loro due. La Colonna, prima del sodalizio con Michelangelo, aveva fatto parte di diversi ambienti riformisti, avendo anche partecipato alle discussioni dirette da Juan de Valdés a Napoli nei primi anni $1530^{2}$. 
Dopo la morte del Valdés, avvenuta nel 1541, il circolo da Napoli si trasferì a Viterbo, ricovero prediletto della Colonna, e il cardinale inglese Reginaldo Polo assunse il ruolo di guida spirituale lasciato vuoto dalla scomparsa del teologo spagnolo. Lì il Polo convinse la Colonna a credere nella salvezza per la fede e ad agire comunque come se la salvezza dipendesse dalle sole opere. Questa convinzione, per tramite della Colonna, doveva fornire le basi del 'nicodemismo' michelangiolesco. Michelangelo e Vittoria Colonna non arrivarono mai ad un'aperta rottura con Roma ma preferirono professare nell'intimo ciò che credevano la giusta fede. Mentre la Colonna, dopo il 1542, intimorita dall'Inquisizione, si distanziò da quella temperie spirituale nonché dai suoi amici convertiti al protestantesimo, Michelangelo rimase fedele alle sue idee, rinchiudendosi in un riformismo cattolico individuale simile a quello professato da Polo a Viterbo e che veniva chiamato "nicodemismo" (De Tolnay, Art and Thought 68) ${ }^{3}$. Pertanto, l'influenza delle idee di Juan de Valdés nella poesia di Michelangelo è inequivocabile. Particolarmente nell'ultimo periodo della vita del fiorentino l'aderenza alle dottrine della giustificazione per la fede e della grazia divina come unica fonte di salvezza si ripresentano spesso nel canzoniere. Si constata, per dirla con Gandolfo, una "consonanza con le motivazioni riformistiche . . . una direzione più fondatamente mistica, di totale abbandono alla forza rigenerante del richiamo divino, di contro alla delineazione di un valutato percorso spirituale" (151).

Clements, discutendo della fede di Michelangelo, fa presente che questi andava a Messa; afferma inoltre che ciò non trova riscontro nelle Rime (42). Tuttavia l'obbedienza ai riti della Chiesa non inficia la nostra tesi; anzi mette in risalto la duplicità di intenzione di Michelangelo consistente nel divario fra la fede esteriore e quella interiore. Nei versi le confessioni dell'artista avvengono senza alcuna mediazione clericale. Difatti il 'nicodemismo' di Michelangelo consisteva in una sorta di resistenza passiva; obbediva ai riti della Chiesa, ma nel medesimo tempo osservava nell'intimo ciò che per lui era la giusta fede. Egli non poteva né voleva conformarsi a nessuna delle diverse tendenze religiose in lizza; quindi non voleva sopprimere l'esigenza interna di forgiare un credo idiosincratico, il quale potesse attingere ad elementi ora riformistici, ora controriformistici.

De Tolnay (Art and Thought 4) nota che il 'repubblicanesimo' di Michelangelo non si estendeva agli strati popolari, che non pagavano le "gravezze". Nello stesso modo traspare nei versi del Buonarroti l'idea che la cognizione del Divino non poteva essere accessibile a tutti, ma ai pochi spiriti eletti. Di conseguenza le grandi masse non figurano nel monologo spirituale del canzoniere michelangiolesco. L'egregio artista non si pone il problema della salvezza altrui, ma rimane coinvolto nella problematica del proprio rapporto con l'aldila ${ }^{4}$.

Difatti, per l'io poetante di Perché Febo non torce e non distende (1-4), il "vulgo" non capisce la vera natura delle tenebre notturne. La debole notte, dal canto suo, esiste in rapporto di complementarietà con il sole, benché essa sia indifesa dinanzi alla luce. Per l'io anch'essa è un sole, sebbene questo fatto sfugga ai più. Nelle quartine di questo sonetto l'io poetante raffigura metafori- 
camente l'incapacità della notte di difendersi con due lunghi sintagmi che mettono a contrasto il lume solare con quello notturno. Il nume, svegliandosi la mattina, stende inconsapevolmente le braccia intorno alla Terra, infrangendo l'integrità della notte. Questo concetto viene rafforzato dalle assonanze e rime interne le quali si insinuano per tutta la poesia ("torce", "intorno", "torchio"; poi "debol", "col", "vuol", "lucciola", "sol"). L'aspra allitterazione di "vulgo volle" (v. 3) ci avverte subito che l'antitesi della luce non è la notte, ma l'ignoranza; questo effetto viene intensificato ulteriormente dalle rime baciate dei versi $2-3$ e 6-7. Il concetto è approfondito nella prima terzina (dove si recupera dal Ficino il rapporto gerarchico fra il Sole, la Terra e la Luna), ma pare che il medesimo io poetante non sappia descrivere con precisione la sua esperienza notturna. Piuttosto si accontenta di imboccare una "via negativa" per definirla poi in termini spregiativi. La terzina conclusiva, anzi, nega il valore della notte in contrasto con quanto è affermato sia nella prima quartina di Perché Febo non torce $e$ non distende sia in $O$ notte, o dolce tempo, benché nero.

Quest'ultimo sonetto, particolarmente nelle terzine, non è un componimento innovativo; ripete piuttosto i luoghi comuni della tradizione petrarchesca. Tuttavia, in $O$ notte, o dolce tempo, benché nero la notte non è così passiva come in Perché Febo non torce e non distende: un novero di verbi di voce attiva ("assalta", "mozzi", "tronchi", "rendi", "rasciughi”, "posi”, "furi”) la caratterizza. Paradossalmente, l'immagine della notte resa qui, quella di un periodo di riposo dopo una giornata lavorativa e metaforicamente dopo la vita terrena, è consona con il ritmo pacato dell'intero componimento ${ }^{5}$.

In Colui che fece, e non di cosa alcuna non si crea un rapporto antinomico fra il giorno e la notte; si ricalca piuttosto una falsariga ficiniana. Come in Perché Febo non torce e non distende, qui il giorno e la notte sono posti in un rapporto di supplementarietà perché entrambi furono creati dallo stesso elemento, il tempo. Il poeta cerca di approfondire questo concetto nel primo emistichio del terzo verso del sonetto dove il ritmo sincopato (causato dalla ripetizione della "d": "d'un due diè") costringe il lettore a fermare l'attenzione sul passo e notare la comune genesi delle due divisioni temporali. La predestinazione bandì l'io del sonetto all'oscurità, quindi egli dovrà cercare la sua salvezza lì. Nella prima terzina l'io poetante (come quel buio notturno attenuato dalla luce lunare e quindi di una oscurità non sufficientemente profonda) cerca di correggersi, tramite la penitenza, ma il suo sforzo, come l'oscurità della notte, è debole e non prevale. L'ultima terzina sviluppa ulteriormente il concetto neoplatonico delle anime gemelle già enunciato nella prima quartina: l'io poetante vorrebbe "far giomo chiar sua oscura notte al sole" dell'oggetto dell'amore sublimato e anagogico. Il desiderio di consegnare alla carta dei versi volutamente difficili i quali tentano di rispecchiare la lotta interiore del poeta è testimoniato qui dalla giustapposizione di bene e male nell'undicesimo verso. "Male", posto al centro dell'endecasillabo e accanto a "bene", acquista un doppio valore semantico. In questo modo possiamo constatare il conflitto dentro chi crede di pentirsi "male" 
("mal m'affliggo e lagno") nonché di chi crede inadeguate le proprie opere pie ("ond'io di far ben mal").

Se chi "esalta" e "onora" la notte ha "intelletto intero", come afferma l'io di $O$ notte, o dolce tempo, benché nero, l'io di Ogni van chiuso, ogni coperto loco "ben vede e ben intende" perché in questo componimento la rigenerazione spirituale dell'uomo si realizza nell'oscurità. Il giorno e la notte diventano metafore di modi alternativi di accedere al divino. Tuttavia, a differenza degli altri sonetti alla notte, qui la superiorità della notte è decisiva. Come Perché Febo non torce e non distende, Ogni van chiuso, ogni coperto loco è un monologo. Quindi, l'io è privo di un interlocutore a cui rivolgersi, ma, ed è ciò che più conta, anche la notte è diversa. A differenza degli altri componimenti del canzoniere michelangiolesco, la passiva luna (di Veggio co' be' vostr'occhi un dolce lume, per esempio [v. 12: "come luna da sé sol par ch'io sia"]), la quale esiste solo in quanto è capace di rispecchiare la luce solare, cede nei sonetti sulla notte ad una concezione divinizzante dell'oscurità ("l'ombra sol a piantar l'uomo serve"). Infatti, il dodicesimo verso è volutamente ambiguo, data la giustapposizione dei glossemi "ombra" e "sol". L'ambivalenza intensifica a sua volta il valore spirituale della notte: è ombra soltanto oppure un'ombra-sole (un'ombra illuminante) che serve a "piantar l'uomo". Pertanto l'io poetante non è visto quale anello nella catena universale dell'Amore ma in qualità di uomo universale, solitario beninteso, il quale contempla la propria vita interiore e la funzione dell'Uomo nel cosmo.

Ogni van chiuso, ogni coperto loco (dei quattro sonetti sulla notte quello dal più felice esito artistico $)^{6}$ s'inizia con una lunga frase la quale trova il suo verbo solo nel terzo verso. In questo modo si ribadisce nella sintassi l'ubiquità della vita spirituale sottolineando nel contempo il valore della fede interiore. Riconoscendo la santità della notte (v. 13) quale metafora di quella spiritualità intima a scapito della sottomissione ai riti di precetto di qualsiasi chiesa, si pone in risalto l'effettiva superfluità di luoghi pubblici di venerazione. La seconda quartina approfondisce l'argomento dei versi 5-8 di Perché Febo non torce e non distende riguardo alla fragilità della notte: non solo un nume come il sole, ma addirittura un verme qualsiasi può aggredire l'integrità della notte. Qui constatiamo il modo in cui l'uso di un'immagine quale quella del verme dimostra quanto l'affanno religioso di Michelangelo prendesse il sopravvento nelle opere più personali $\mathrm{e}$ risentite spazzando via ogni pretesa di aderenza o di conciliazione con le norme petrarcheggianti tipiche dell'epoca ${ }^{7}$.

Per l'io poetante di Ogni van chiuso, ogni coperto loco la materia più banale vegeta al sole (vv. 9-11), ma alla notte è riservata l'antimateria, l'etereo. La logica conclusione del discorso, il "dunche" (che riecheggia l'indeterminato "quantunche" del secondo verso) di un soliloquio quasi mistico, atto a sfidare la logica, culmina sottolineando la divinità della vita interiore e contrapponendola all'ostile cosmo. Il dodicesimo verso è volutamente ambiguo ed evoca Paolo, il quale nella cecità ricevette il lume della sapienza divina. Nell'ultimo verso, là 
dove ci sarebbe da aspettarsi il glossema "quando" come termine conseguente del rapporto temporale avviato nel tredicesimo verso, il pur minimo cambiamento di un fonema (si muta l'attesa dentale sonora di "quando" nell'inattesa dentale sorda di "quanto") aumenta il senso di incertezza e di ambiguità. Non ci troviamo di fronte, quindi, ad un argomento logico conclusivo, bensì ad un'esclamazione alogica riguardante l'intrinseca divinità dell'uomo il quale ha un cuore (un "coperto loco") inerme, ma ciononostante (e forse proprio per questo) in grado di rendergli accessibili le porte del Paradiso.

Dunque le immagini di $O$ notte, o dolce tempo, benché nero rientrano nella tradizione petrarchesca, laddove quelle di Perché Febo non torce e non distende e di Colui che fece, e non di cosa alcuna ricalcano il Convito di Platone di Marsilio Ficino. In Perché Febo non torce e non distende la notte "è figlia del sol e della terra" e quindi si appropria del lume solare per dare a sua volta alla terra la propria l'oscurità: concetti mutuati dalla "IV Orazione" di Sopra lo Amore. In Colui che fece, e non di cosa alcuna "l'amore nasce da similitudine" 8 e permette all'io di ricercare "quel lume soprannaturale, che già fu l'altro mezzo dell'anima" (Ficino 50). Ogni van chiuso, ogni coperto loco, invece, si distingue dagli altri sonetti sulla notte in quanto è l'oggetto stesso del discorso poetico, la notte, ad assumere un significato diverso. Completamente assente da questo sonetto è l'idea ficiniana dell'amore anagogico, soppiantato da una qualità affatto intrinseca all'uomo.

Il valore dei versi di Michelangelo va ben oltre il mero sperimentalismo poetico; si riscontra nella tensione etico-ideale e nella lotta esasperata del poeta contro le proprie inadeguatezze in nome di un ideale non solo di perfezione artistica ma anche e soprattutto in nome di un'aspirazione spirituale refrattaria ad ingerenze esterne ${ }^{9}$. In Ogni van chiuso, ogni coperto loco la notte, metafora della vita individuale ed interiore, dà all'uomo la sua dignità: la sua capacità di rendersi divino.

Michigan State University

\section{NOTE}

1 Sarà necessario in altra sede considerare questi sonetti sulla notte come parte del gruppo di versi dedicati alla stampa nel contesto delle affinità politiche di Michelangelo ai fuorusciti fiorentini a Roma, particolarmente coloro che lo coadiuvarono con la preparazione del florilegio, Luigi del Riccio e Donato Giannotti. De Vecchi distingue fra "rime composte all'incirca tra il 1532 e il ' 47 . . . indirizzate a persone quali il Cavalieri o la Marchesa di Pescara, per le quali l'artista nutriva profonda devozione e reverenza, o erano comunque destinate a far parte del gruppo scelto per l'edizione, cioè a divenire in un certo senso pubbliche" e quelle anteriori e posteriori a quelle date, scritte per "sfogo personale" (386-87).

2 La Colonna strinse amicizia, a Napoli, con Valdés e i suoi accoliti, poi conobbe Michelangelo e nel 1537 iniziò un soggiorno alla corte di Renata di Francia (Giovanni Calvino ne era appena partito) che doveva prolungarsi più di un anno (Reumont 158-171). Valdés professava delle dottrine simili a quelle di Lutero e di Calvino benché non avesse avuto 
contatti diretti con i due leader del protestantesimo settentrionale. Valdés, come Lutero e Calvino, credeva che l'individuo non avesse bisogno di un intermediario per la salvezza. Non credeva nell'infusio gratiae dei sacramenti, ma dava importanza soltanto al rapporto fra l'anima e il sacrificio di Cristo: la grazia, condizione indispensabile per la redenzione, dipendeva esclusivamente dalla fede. Nei suoi insegnamenti Valdés insisteva, tra l'altro, sulla giustificazione attraverso la sola fede, recuperata dalla lettura di Paolo (per Valdés vedi Nieto; Cantimori, Umanesimo 193-203). Vedi altresì Lucente, "Lyric Tradition" (321 e sgg.) per l'importanza della lettura di Paolo nonché per l'irrisolta "crisi di volontà" in Michelangelo.

3 Per nicodemismo si intende un movimento evangelico di riformisti rimasti in patria durante il periodo della Riforma e della Controriforma. Per i nicodemiti contavano solo la fede interiore e le opere. A riguardo vedi Cantimori, Prospettive 27-66; secondo Cantimori "la prassi nicodemitica diventa così perfetta che non lascia traccia di sé" (29). Tuttavia si intravede l'affinità tra l'evangelismo nicodemitico e quello di Valdés, il quale sempre secondo Cantimori - "dimostrava indifferenza alle discipline e alle forme ecclesiastiche, indifferenza alle questioni dogmatiche, desiderio dell'unità fra tutti i cristiani sulla base della semplice morale evangelica (imitazione di Cristo)" (33).

4 Cambon (66) scrive di un "passionate introvert who hid so much in himself that he could convey only through the symbols of art".

5 Per vedere l'affinità fra questo sonetto e la tradizione petrarchesca si può paragonarlo al dellacasiano "O sonno, o de la queta, umida, ombrosa". Cfr. altresì Gandolfo (119-21).

6 Pertanto, benché $O$ notte, o dolce tempo, benché nero sia fra i componimenti michelangioleschi più conosciuti, secondo Girardi solo Ogni van chiuso, ogni coperto loco e Colui che fece, e non di cosa alcuna erano destinati alla stampa.

7 Questa problematicità religiosa, inoltre, doveva rimpiazzare in Michelangelo il desiderio affatto terreno di incidere politicamente in prima persona nella vita democratica della repubblica fiorentina, sconfitta prima nel 1530 dalle forze di Carlo V e Clemente VII e poi in modo definitivo da Cosimo I nel 1537. Prova poetica dei profondi sentimenti repubblicani dell'artista sono l'epigramma Caro m'è'l sonno e più l'esser di sasso e i sonetti Dal ciel discese, e col mortal suo e Quante dirne si de' non si può dire (dedicati ad un altro famoso esule fiorentino: Dante Alighieri), il madrigale sulla tirannia nonché il busto del tirannicida Bruto (del 1540). Per la datazione dei sonetti rinvio all'apparato dell'edizione delle Rime curata da Girardi (283-85). La fede di Michelangelo traspariva nei suoi versi ed anche in quello che dipingeva per Paolo III. Non a caso il sodalizio con la Colonna, il Giudizio universale, l'affresco della conversione di Saulo e i sonetti sulla notte appartengono tutti alla medesima decade, iniziata nel 1536. La Pietà del Duomo (un gruppo marmoreo dove appare l'autoritratto buonarrotiano sul volto di Nicodemo, "l'apostolo clandestino" e simbolo del riformismo cattolico post-tridentino), del quinquennio immediatamente successivo alla morte della Colonna, è di poco posteriore a queste opere. De Tolnay fissa la data dell'esecuzione dell'affresco di Saulo fra il 1542 e il 1545; a suo parere Michelangelo lavorò sulla Pietà del Duomo fra il 1547 e il 1553. Michelangelo intendeva far porre la Pietà del Duomo sulla propria sepoltura, ma abbandono il marmo; lo sfregio dopo le ripetute insistenze dell'aiutante Urbino di portarlo a compimento (Michelangelo 70, 86-88). Ha larga risonanza nel Giudizio un'iconografia tutt'altro che ligia all'ortodossia controriformistica. Le anime sono sole con il loro creatore; né Maria né i santi possono intervenire a loro favore presso il severo arbitro. Michelangelo raffigura un Creato tetro, senza natura, dove la misericordia di Dio è tanto terribile quanto la sua condanna. Ma ciò che più riguarda la tesi che andiamo postulando è la rielaborazione di questo affresco operata dal Buonarroti. Nelle prime versioni la Madonna capeggiava gli eletti al Paradiso; nell'opera presentata al pubblico essa si nasconde nel suo manto mentre un'umanità indifesa si dirige verso la figura dominante di Cristo. 
8 Cfr. la "II Orazione" di Sopra lo Amore.

9 Si ricordi il lettore dei sonetti inviati al Vasari nei quali si esalta chi sa imporre la propria volontà alla Natura, nonché Com'esser, donna, può quel c'alcun vede, scritto per la Colonna.

\section{OPERE CITATE}

Buonarroti, Michelangelo. Rime con Varianti, Apparato, Nota filologica. Ed. Enzo Noè Girardi. Bari: Laterza, 1960.

Cantimori, Delio. Prospettive di storia ereticale italiana nel Cinquecento. Bari: Laterza, 1960. . Umanesimo e religione nel rinascimento. Torino: Einaudi, 1975.

Cambon, Glauco. Michelangelo's Poetry. Princeton: Princeton UP, 1985.

Clements, Robert J. The Poetry of Michelangelo, New York: New York UP, 1966.

De Tolnay, Charles. The Art and Thought of Michelangelo. Trans. Nan Buranelli. New York: Pantheon, 1964.

. Michelangelo. Vol. 5. Princeton: Princeton UP, 1960.

De Vecchi, Pier Luigi. "Studi sulla poesia di Michelangelo". Giornale storico della letteratura italiana 140 (1963): 363-402.

Ficino, Marsilio. Sopra lo amore o ver' Convito di Platone. Comento di Marsilio Ficini fiorentino sopra il Convito di Platone. Ed. G. Ottaviano. Milano: Celuc, 1973.

Gandolfo, Francesco. "Sogno, orfismo e motivazioni riformistiche nell'opera di Michelangelo". Il "dolce tempo" : mistica, ermetismo e sogno nel Cinquecento. Roma: Bulzoni, 1978. 113-58.

Lucente, Gregory L. "Lyric Tradition and the Desires of Absence: Rudel, Dante and Michelangelo ('Vorrei uoler')". Canadian Review of Comparative Literature 10.3 (September 1983): 305332.

Nieto, Josè C. Juan de Valdés and the Origins of the Spanish and Italian Reformation. Geneva: Droz, 1970.

Reumont, Alfred von. Vittoria Colonna: vita, fede poesia nel secolo decimosesto. Torino: Loescher, 1892.

Saslow, James M. The Poetry of Michelangelo. New Haven: Yale UP, 1991. 